\title{
Specificity of Circadian Function in Transplants of the Fetal Suprachiasmatic Nucleus
}

\author{
David J. Earnest, Celia D. Sladek, Don M. Gash, and Stanley J. Wiegand \\ Department of Neurobiology and Anatomy, University of Rochester School of Medicine, Rochester, New York 14642
}

Fetal tissues obtained from specific regions of the developing hypothalamus were transplanted to determine whether the precursor neurons of the suprachiasmatic nucleus (SCN) can be distinguished from those of the presumptive paraventricular nucleus (PVN) on the basis of the functional capacity to generate circadian rhythms. The presumptive SCN, the PVN, and a portion of the neocortical primordium were dissected from the developing forebrains of normal Long-Evans fetuses, separated, and selectively transplanted into the periventricular-third ventricle region of adult, vasopressin (VP)-deficient Brattleboro rats. In host animals that received grafts containing the precursor population of SCN neurons, the temporal profile of VP levels in the cerebrospinal fluid (CSF) oscillated with a circadian periodicity in a manner similar to that observed in normal Long-Evans rats. CSF collected serially from animals with grafts of the presumptive PVN also contained VP, but no circadian variation was manifested in peptide levels. VP was undetectable in CSF samples obtained from Brattleboro rats with cortical grafts. In association with their circadian functional capacity, grafts of the SCN primordium were characterized by clusters of parvicellular neurons immunopositive for VP or vasoactive intestinal polypeptide (VIP) that resembled the cell groups of the in situ SCN. In contrast, transplants of the presumptive PVN did not contain neurons immunoreactive for VIP, and the VP neurons in these grafts resembled the neurosecretory cells of the PVN. These results demonstrate that grafts containing VP neurons derived from the primordial SCN develop not only the cytological and neurochemical features which distinguish this population from the VP neurons of the PVN in situ but also the unique functional capacity of a circadian pacemaker to generate endogenous rhythms in secretory activity.

The suprachiasmatic nucleus (SCN) of the hypothalamus is an integral neural locus for the generation of circadian rhythms in mammals. Recent applications of the neural transplantation technique to the study of the mammalian circadian organization have served to corroborate evidence for this function of the SCN. Specifically, transplants of fetal hypothalamus containing the SCN have been reported to restore circadian rhythms in bchavioral activities of SCN-lesioned hosts, such as drinking

\footnotetext{
Received July 29, 1988; revised Nov. 8, 1988; accepted Jan. 18, 1989.

Correspondence should be addressed to David J. Earnest, Department of Neurobiology and Anatomy. University of Rochester School of Medicine, $601 \mathrm{Elm}$ wood Ave., Rochester, NY 14642.

Copyright $\odot 1989$ Society for Neuroscience $0270-6474 / 89 / 082671-07 \$ 02.00 / 0$
}

and locomotor activity (Drucker-Colin et al., 1984; DeCoursey and Buggy, 1986; Lehman et al., 1987). However, little is known about the mechanisms by which these transplants restore rhythmicity. One possibility is that the grafted neurons restore rhythmicity by providing a specific trophic factor(s) which induces plasticity in the neural organization of the circadian system and enables other neurons in the host brain to assume this timekeeping function in the absence of the in situ SCN. Alternatively, the transplanted hypothalamic neurons may intrinsically function as a circadian pacemaker and restore rhythmicity in SCNlesioned hosts through neurohumoral signals and/or the establishment of neural connections with the host brain. In this regard, if the transplanted neurons are functioning as a circadian pacemaker, then endogenous biological activities expressed by these neurons should oscillate with a circadian periodicity. This hypothesis is based on observations that indices of SCN activity such as neuronal firing rate and secretory activity oscillate in a circadian fashion and that the oscillatory nature of these activities is an endogenous feature of SCN neurons (Green and Gillette, 1982; Inouye and Kawamura, 1982; Earnest and Sladek, 1987; Gillette and Reppert, 1987). Consequently, the present investigation was conducted to determine whether grafts of the primordial SCN develop the distinctive capacity to function as a circadian clock.

Since the circadian patterns of vasopressin (VP) secretion observed in vivo and in vitro (Schwartz and Reppert, 1985; Earnest and Sladek, 1987; Gillette and Reppert, 1987) appear to directly reflect the intrinsic activity of a prominent population of VP neurons located in the SCN (Vandesande et al., 1975; Moore, 1983), the present study utilized VP release as an index of the activity of transplanted SCN neurons. Experiments were designed to determine whether grafts containing the precursor populations of SCN neurons develop the capacity to release VP into the cerebrospinal fluid (CSF) in a circadian fashion. Brattleboro rats were utilized as transplant recipients because the genetic deficiency in brain VP in this strain (Sokol et al., 1976) allows unequivocal identification of vasopressinergic neural processes and VP in the CSF as emanating from grafts of normal tissue.

Based on reports indicating that grafts of the fetal anterior hypothalamus frequently contain 2 or more cytologically distinct populations of VP neurons that resemble the normal complements found in the in situ paraventricular, supraoptic and suprachiasmatic nuclei (Boer et al., 1985; Wiegand and Gash, $1987,1988 \mathrm{a})$, this study was designed to provide for the transplantation of a more homogeneous population of SCN-like VP neurons. Our approach entailed the selective transplantation of topographically discrete regions of the developing hypothalamus 
containing the anlagen of either the $\mathrm{SCN}$ or paraventricular nucleus (PVN; Altman and Bayer, 1978a, b) so as to evaluate specificity in the capacity of the grafts to generate circadian VP rhythms. The relationship between the functional capacities and the specific cytological and immunohistochemical features of grafts was also evaluated.

\section{Materials and Methods}

Animals and housing conditions. Young adult male rats of the Brattleboro strain (Blue Spruce Farms), homozygous for diabetes insipidus, were utilized as transplant recipients. Age-matched male Long-Evans rats (Charles Rivers Laboratories) served as nontransplanted controls. Throughout this study, experimental and control animals were housed individually and maintained in a temperature-controlled environment $\left(21^{\circ} \mathrm{C}\right)$ with food and water provided ad libitum. Except where noted otherwise, the animals were exposed to a 12-hr:12-hr light-dark cycle (lights on 06.00 to 18.00). Donor tissue of known gestational age was obtained from the fetuses of Long-Evans females (Charles Rivers Laboratories) that had been bred with males of the same strain. The pregnancies were timed by considering the morning of sperm detection in the vaginal lavage as day 0 of gestation.

Preparation and transplantation of donor tissue. Fetuses were removed from Long-Evans dams, killed on days 15, 16, or 17 of gestation and placed in an ice-cold chamber moistened with Eagle's medium. The fetal brain was removed and a coronal section containing the anterior hypothalamus was obtained by making incisions just anterior to the optic chiasm and at the midpoint between the chiasm and the developing mammillary bodies. Using topographical landmarks described by Altman and Bayer (1978a, b), the presumptive anlagen of the SCN and PVN were selectively dissected from the coronal section by excising blocks of tissue located immediately dorsal to the midline portion of the optic chiasm and adjacent to the dorsal dilation of the third ventricle, respectively. In addition to this dissection of hypothalamic tissue, an equal volume of developing cortical tissue was removed from the coronal sections just dorsal to the lateral ventricle. Each block of donor tissue was minced into small pieces and maintained in Eagle's medium on ice.

Immediately prior to transplantation, the minced SCN, PVN, or cortical tissue from a single fetal donor was drawn into a 20 -gauge spinal needle mounted on a stereotaxic instrument. Using coordinates from the brain atlas of Paxinos and Watson (1986), the cannula was positioned in the periventricular-third ventricle region of anesthetized recipients (chloral hydrate; $42 \mathrm{mg} / \mathrm{kg}$ body weight) and the tissue was slowly ejected. All fetal tissue was transplanted within $1 \frac{1}{2} \mathrm{hr}$ after death of the dam. A total of 48 animals received periventricular-third ventricle grafts of fetal hypothalamic or cortical tissue.

CSF collection and radioimmunoassay of VP concentrations. Using the method described by Schwartz and colleagues (1983), a 21-gauge cannula was implanted 6 to 12 weeks post-transplantation into the cisternae magna of transplant recipients and Long-Evans controls. Following completion of this surgery, animals were allowed to recover for 24-36 hr prior to experimentation.

Lighting conditions were then changed from LD 12:12 to constant light ( $\sim 150$ lux $)$, and samples of CSF were serially collected from individual unanesthetized animals at 6-hr intervals (i.e., 06.00, 12.00, 18.00 , and $24.00 \mathrm{hr}$ ) for $1-5 \mathrm{~d}$. At each sampling interval, 125-150 $\mu \mathrm{l}$ of CSF was removed from the cisternae magna by using a small syringe to draw the CSF into a length of polyethylene tubing capable of harboring the entire volume of the sample. All CSF samples were immediately dispensed, stored at $5^{\circ} \mathrm{C}$ for a few hours, and centrifuged for $10 \mathrm{~min}$. The CSF was separated from its pellet, if any, and samples showing any signs of blood contamination following this separation were excluded from further analysis. All samples were frozen and stored at $-40^{\circ} \mathrm{C}$. VP concentration in duplicate 50- $\mu$ l aliquots of each CSF sample was measured by radioimmunoassay (Sladek et al., 1986) using an antibody provided by Drs. Lindheimer and Durr of the University of Chicago. The minimum sensitivity of the assay was $0.1-0.5 \mathrm{pg} /$ tube, and crossreactivity with oxytocin was less than $0.001 \%$. Interassay variation was $15.6 \%$. VP levels in all samples from any given animal were measured in a single assay.

To evaluate the temporal pattern of VP in the CSF for circadian variation, analysis focused on the sampling interval during which VP levels reached a peak value based on the criterion that this value was at least 2 times greater than the nadir value for the cycle. Using this phase reference point, the time interval between consecutive reference points was measured so as to determine period length and, ultimately, whether the peak in CSF VP recurred with a circadian periodicity. Statistical analysis was performed on the means for VP levels using a one-way analysis of variance with repeated measures to determine the significance of sampling time. Group means in this analysis were obtained by arbitrarily aligning the highest value for CSF VP in individual animals because the individual profiles expressed by SCN (or PVN) grafts were not phase-coordinated with each other. Differences in VP levels between sampling intervals within a given circadian day were tested post hoc for significance using the Newman-Keuls sequential range test.

Histological procedures. Following the completion of CSF sampling, animals were killed with a lethal dose of sodium pentobarbital and perfused with fixative solution (4\% paraformaldehyde or $2 \%$ paraformaldehyde and $0.1 \%$ glutaraldehyde), and the brains were sectioned and prepared for immurohistochemical processing as described previously (Watson et al., 1986). A few of the animals with neural grafts were perfused and subsequently injected with opaque gelatin-ink solutions for the visualization of microvasculature (Wiegand and Gash,1988a). A 1 -in- 6 series of $30-\mu \mathrm{m}$ sections was processed for localization of arginine VP. Additional series were processed for localization of vasoactive intestinal polypeptide (VIP) or were stained with thionin. The remaining series of sections were reserved for further immunohistochemical analysis. Peptides were detected by means of an avidin-biotin immunoperoxidase procedure (Watson et al., 1986; Wiegand and Gash, 1988b). The primary antisera against VP (ICN Immunobiologicals) and VIP (ICN Immunobiologicals) were raised in rabbits and used at dilutions of 1:5000-7000 and 1:2000-5000, respectively. The specificity of immunostaining was verified in unoperated Long-Evans and Brattleboro rats by using standard procedural and absorption controls (Wiegand and Gash, 1988b).

\section{Results}

In 24 of the 48 animals with neural grafts, CSF sampling was compromised by either blood contamination or obstruction of the cannula to the extent that the number of samples for a given individual was inadequate to evaluate the temporal profile of VP levels. However, CSF samples were successfully collected for $1-5 \mathrm{~d}$ from the remainder of the grafted animals and from all of the Long-Evans rats.

VP levels in the CSF of nontransplanted Long-Evans controls ranged from $2-17 \mathrm{pg} / \mathrm{ml}$, and in all 3 cases the temporal profile of these VP levels fluctuated with a circadian periodicity. As shown in Figure 1, the circadian patterns of VP in the CSF of these animals were phase-coordinated by the presampling lightdark cycle such that all rhythms were characterized by peak VP levels during the interval where the onset of light phase would have occurred in the 12-hr:12-hr light-dark cycle (i.e., $06.00 \mathrm{hr}$ ) and low values throughout the remainder of the circadian cycle. Within a given animal, the peak of the VP rhythm was 2 to 5 times greater than the nadir (mean amplitude of the rhythm = $3.8 \pm 0.4$ ). In the absence of $24-\mathrm{hr}$ light-dark cues, the circadian VP rhythms in Long-Evans controls persisted during the sampling period (i.e., 3-4 d) without any significant damping in the rhythm amplitude.

Similar to those in Long-Evans rats, CSF VP concentrations oscillated on a circadian basis in 7 out of 8 Brattleboro rats that had received grafts containing the anlage of the SCN (mean period $=24.0 \pm 0.0 \mathrm{hr}$ ). In addition, the VP rhythms in animals with SCN grafts were similar in amplitude (2- to 7 -fold decrease from peak to nadir; mean $=3.7 \pm 0.4$ ), and in the range of CSF peptide levels (1-22 pg/ml). Values for VP levels during the peak of the rhythm were significantly greater $(F=14.00 ; p<$ $0.01)$ than the values for all other sampling intervals during a given circadian day. Unlike those in Long-Evans controls, the VP rhythms in SCN-grafted animals were not phase-coordi- 

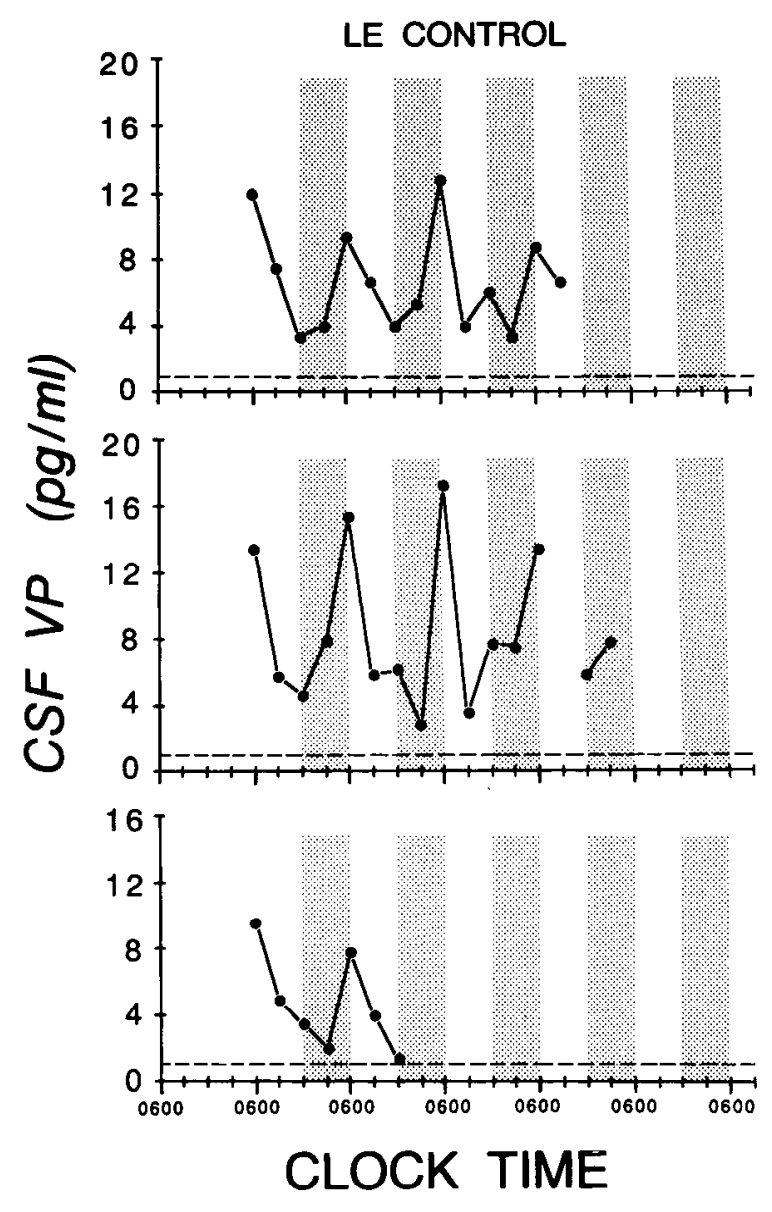

Figure 1. Temporal profiles of CSF VP for 3 individual Long-Evans rats. All animals were studied in constant light, and CSF was collected at 6-hr intervals. Dashed lines indicate the minimum VP concentration detectable in sample aliquots by radioimmunoassay. Graphs are stippled to aid in the visualization of the VP rhythms, with the stippled areas representing the subjective night of the circadian cycle. Specific lighting conditions prior to and during the period of CSF sampling are indicated at the bottom, with the hours of light and dark represented by open and closed bars.

nated by previous exposure to a light-dark cycle (Fig. 2). Furthermore, the individual rhythms expressed by $\mathrm{SCN}$-grafted animals were not phase-coordinated with each other; timing of the daily peak in VP levels in individuals was not synchronous throughout the group.

VP levels in all CSF samples collected from Brattleboro hosts with grafts of the primordial PVN $(n=8)$ were also comparable to those observed in Long-Evans controls, ranging from 1-14 $\mathrm{pg} / \mathrm{ml}$. However, variations in peptide levels were insignificant and irregular rather than circadian in nature (Fig. 3). Moreover, the maximum daily fluctuation in VP levels in PVN-grafted animals (mean $=3.0 \pm 0.7 \mathrm{pg} / \mathrm{ml})$ was significantly less $(t=$ $3.05 ; p<0.01)$ than the fluctuations observed in either LongEvans controls $(9.6 \pm 0.3 \mathrm{pg} / \mathrm{ml})$ or SCN-grafted animals $(7.6$ $\pm 0.9 \mathrm{pg} / \mathrm{ml})$. In Brattleboro rats with grafts of the fetal neocortex $(n=8)$, VP levels in all CSF samples were below the limits of detection.

Viable grafts were identified in all host animals. The transplanted tissue was generally organized into a single body or 23 large aggregations located in the host periventricular hypo-
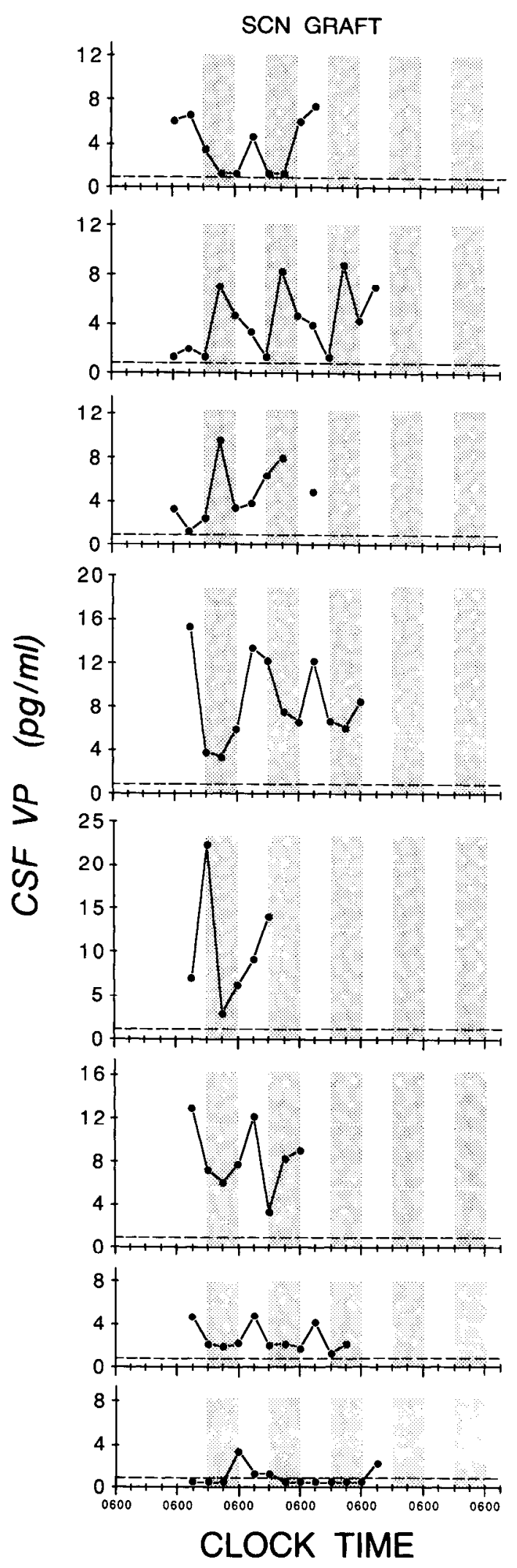

Figure 2. Patterns of CSF VP for individual Brattleboro rats with grafts of the presumptive SCN $(n=8)$ studied in constant light. 

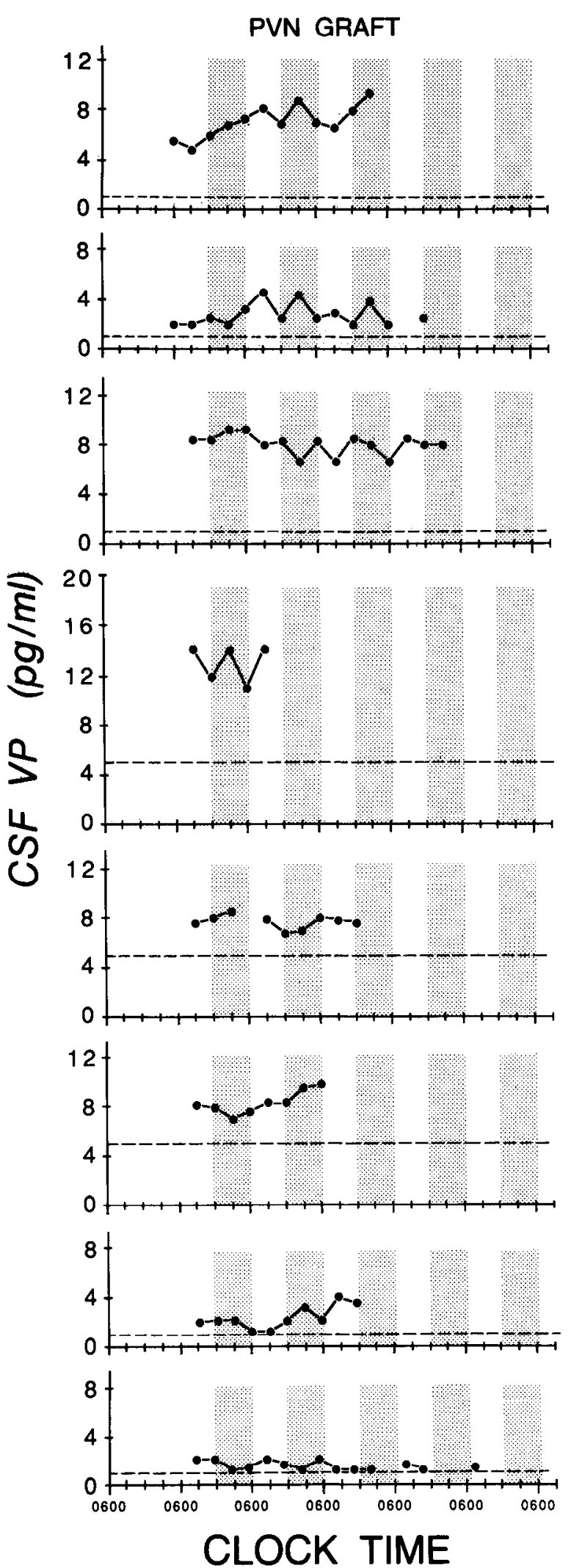

terns of CSF VP for individual Brattleboro rats with grafts Figure 3. Patterns of CSF VP for individual Brattleboro rats
of the presumptive PVN $(n=8)$ studied in constant light. thalamus and/or third ventricle. Vascular inosculation of the transplants consistently occurred in areas where the host brain and graft were directly apposed. Many grafts werc characterized by a considerable increase in size following transplantation such that the ventricular lumen was partially occluded or, in a few cases, a portion of the graft extended through the ventral surface of the host brain. However, host neural structures in the vicinity of the graft appeared to experience little damage as a result of the transplantation procedure or graft proliferation.

Identification of immunoreactive elements within the grafts revealed a number of morphological and cytological distinctions between grafts derived from the anlagen of the SCN, PVN, or neocortex. All grafts derived from the primordial SCN contained at least one aggregation of parvicellular neurons immunopositive for VP arranged in an SCN-like fashion (Fig. 4D). The number and relative size of these aggregations were variable among SCN grafts. The perikarya of the VP neurons located in SCN-like cell groups were round or slightly ovoid and were approximately 9-13 $\mathrm{m}$ in diameter (Fig. 5B). VP-immunoreactive fibers of very fine caliber ramified extensively around the cell bodies of origin, establishing a dense local plexus. The vasopressinergic elements within these SCN-type cell groups were invariably associated with a substantial population of neurons immunopositive for VIP that were similar in size and shape (Fig. 4B). The VIP neurons in these clusters generated dense, local arborizations of fibers that were similar to and topographically coincident with the fibers of VP neurons. An appreciable number of VP neurons morphologically distinct from those of the SCN were present in 2 of the SCN grafts. These VP cells were larger and resembled neurosecretory neurons. Moreover, these larger VP neurons were not associated with VIP-immunoreactive neurons and were located outside the boundaries of SCN-like neuronal aggregations.

Neuronal perikarya immunopositive for VP were also identified in grafts derived from the primordial PVN. However, the morphological characteristics of these vasopressinergic neurons more closely resembled those of the neurosecretory cells in the in situ PVN. The perikarya of parvicellular VP neurons were identified in all PVN grafts, but the cells were larger $(12-18 \mu \mathrm{m}$ in diameter) and were somewhat elongated in comparison with those found in SCN grafts (Fig. 5A). These VP neurons were organized into distinct aggregations, but the cells were fewer in number and less densely packed than those in SCN-like clusters (Fig. 4C). Neurites emanating from these VP neurons were often found in direct apposition to blood vessels. In 4 of 8 PVN grafts, VP immunoreactivity was also observed within a small number of neurons of even greater size ( $>20 \mu \mathrm{m}$ in diameter) that resembled the magnocellular neurosecretory cells of the PVN. Unlike the vasopressinergic elements derived from the fetal SCN, the PVN-like neurons in these grafts were never found in association with VIP-immunopositive cells (Fig. 4A). However, a few SCN-like VP neurons were identified in one PVN graft, and these cells in turn were associated with a small number of VIP cells. Four of 8 control grafts of fetal cortex contained VIPimmunoreactive elements, but all were totally devoid of cell bodies and nerve fibers immunopositive for VP.

\section{Discussion}

The present data demonstrate that selective transplantation of the precursor neurons of the SCN and PVN into VP-deficient Brattleboro rats yields grafts retaining the functional capacities that distinguish the in situ SCN and PVN. Functional distinc- 

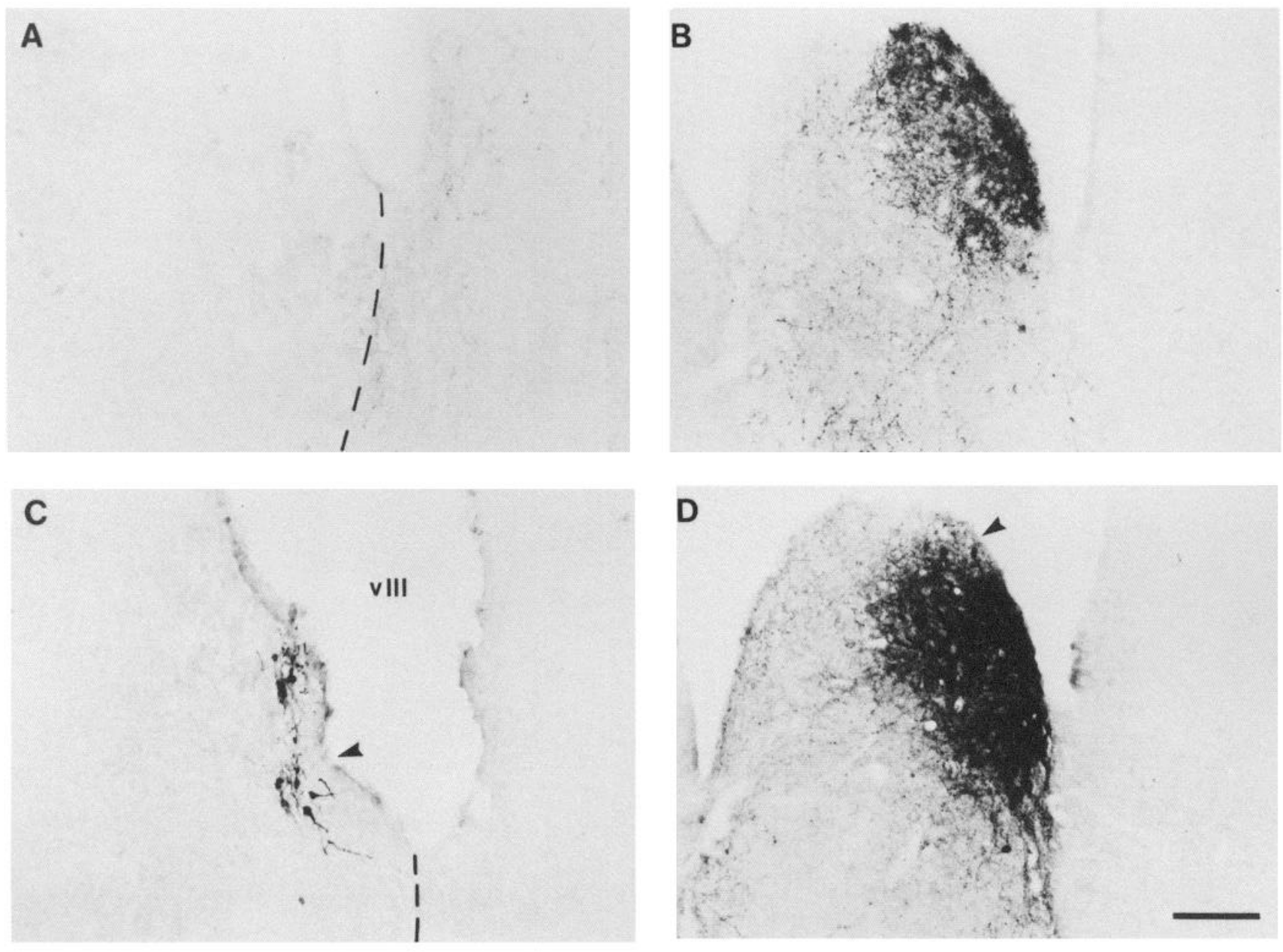

Figure 4. Bright-field photomicrographs of periventricular grafts of the fetal anlagen of the $\mathrm{PVN}(A, C)$ and the $\mathrm{SCN}(B, D)$. Neurons immunopositive for VIP are characteristically absent in a graft of the presumptive PVN $(A)$, but VP-immunopositive neurons resembling the neurosecretory cells of the in situ PVN are localized in the grafted tissue (C). In adjacent sections, the close association of parvicellular neurons immunoreactive for VIP $(B)$ or VP $(D)$ and their arrangement in densely packed aggregations are evident within a graft of the presumptive SCN. Dashed lines in $A$ and $C$ delineate graft-host interface. Arrows in $B$ and $D$ denote regions shown at higher magnification in Figure 5. $v I I I$, Third ventricle. Scale bar, 100 $\mu \mathrm{m}$.

tions between these hypothalamic nuclei have been evidenced previously in work demonstrating that the in situ $\mathrm{SCN}$, but not $\mathrm{PVN}$, is involved in the generation and expression of a circadian VP rhythm in the CSF of Long-Evans rats (Schwartz and Reppert, 1985). Consistent with in situ observations, grafts of the presumptive $\mathrm{SCN}$ were distinguished by the ability to generate circadian rhythms of peptide release. Grafts of fetal PVN, like the endogenous PVN, were capable of producing measurable levels of VP in the CSF, but no circadian variation was manifested in peptide concentrations.

While grafts of the presumptive SCN generated VP rhythms in Brattleboro hosts that were similar in waveform and amplitude to the oscillations expressed in normal Long-Evans rats, the rhythms in SCN-grafted animals and nontransplanted controls were clearly marked by differences in their responses to lighting conditions. In a manner similar to that described previously (Schwartz et al., 1983), the VP rhythms of all LongEvans controls were synchronized by the presampling light-dark environment such that peptide levels peaked in unison at the onset of the subjective day (i.e., projected time during circadian cycle coinciding with onset of light in presampling 12:12 light- dark cycle). In contrast, the rhythms expressed by grafts of the presumptive SCN were not phase-coordinated by previous exposure to the 12:12 light-dark cycle. This observation suggests that regulation of the VP rhythm is not mediated by retinal innervation of the graft or by the in situ circadian system even though SCN-intact Brattleboro rats were utilized as transplant recipients. Instead, the generation of VP rhythms by grafts of the presumptive $\mathrm{SCN}$ probably reflect the rhythmic activity of $\mathrm{SCN}$-like elements within the grafts, suggesting that $\mathrm{SCN}$ grafts are capable of functioning as independent circadian pacemakers. It is also noteworthy that while grafts were obtained from fetuses on day 15-17 of gestation, the earliest sign of a functional circadian clock in the in situ SCN has been detected on gestational day 19 (Reppert and Schwartz, 1984). Thus, the present data further suggest that either circadian activity within the in situ $\mathrm{SCN}$ is initiated at an earlier stage of development or that the fetal anlage of the SCN developed this capacity de novo following transplantation.

The results of the present experiments also provide some insight into the mechanism by which transplants of the fetal hypothalamus restore circadian rhythmicity in SCN-lesioned 

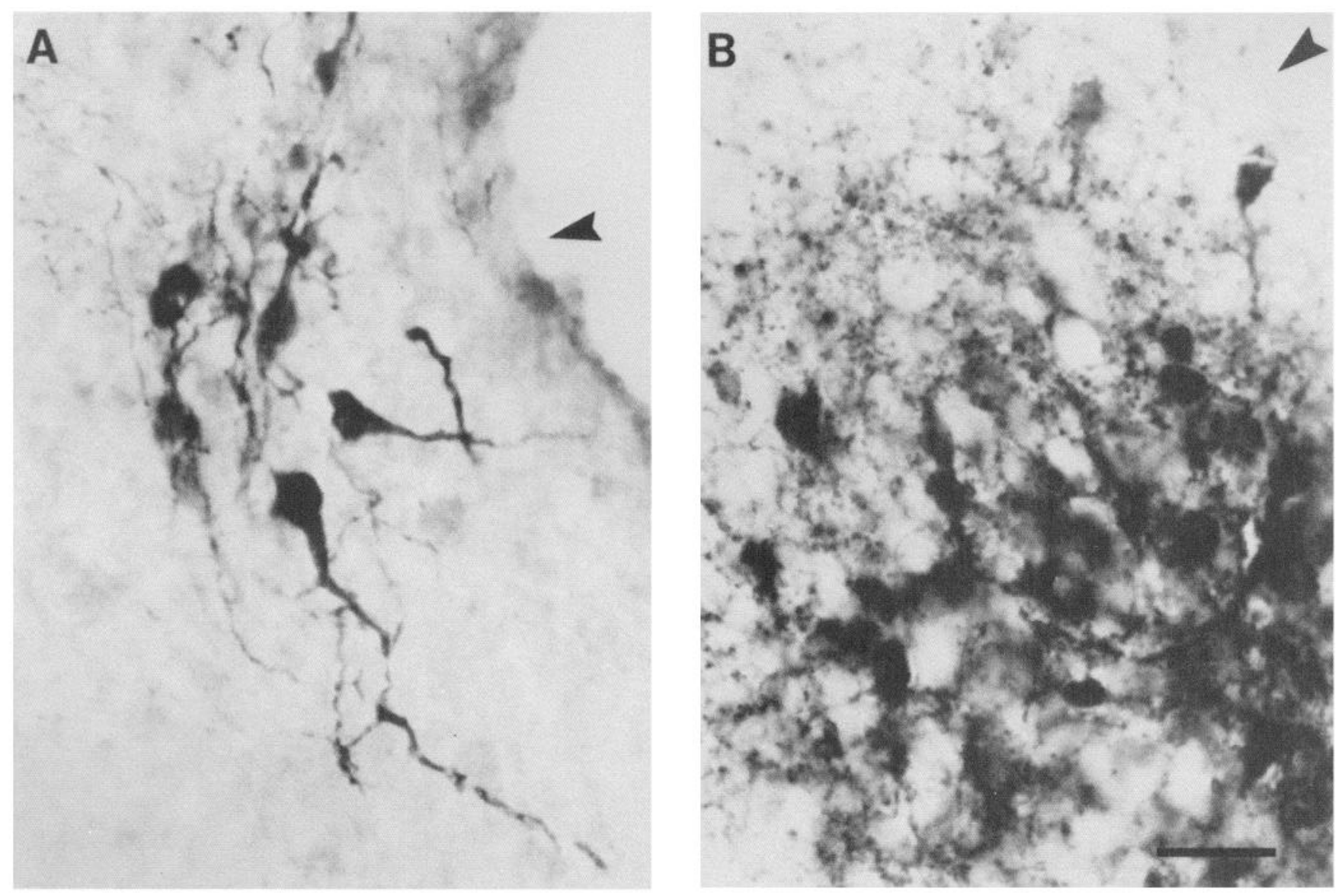

Figure 5. High-power photomicrographs of VP-immunopositive neurons in periventricular grafts of the presumptive PVN $(A)$ and the SCN $(B)$. Arrows provide reference to regions shown at lower magnification in Figure 4. Scale bar, $25 \mu \mathrm{m}$.

hosts (Drucker-Colin et al., 1984; DeCoursey and Buggy, 1986; Lehman et al., 1987). The demonstration of circadian fluctuations in the endogenous secretory activity of transplanted SCN neurons is in accord with the hypothesis that grafts of the developing hypothalamus restore rhythmicity by generating circadian signals that regulate the temporal pattern of various cellular, physiological, and behavioral events. Although the issue of whether these circadian signals were transmitted to the host brain via a neural versus a humoral route remains in dispute, these data indicate that SCN grafts are at least capable of generating circadian humoral signals that could serve to drive rhythms in other processes. Consequently, the finding that SCN grafts express rhythmic secretory activity is consistent with the observations of Lehman et al. (1987) that the restoration of circadian function in SCN-lesioned hosts may occur even in the absence of appropriate neural connections between the graft and the host brain. On the other hand, grafted $\mathrm{SCN}$ has been reported to establish efferent connections with areas of the host brain which normally receive input from the in situ SCN (Wiegand and Gash, 1988b), suggesting that the neural connectivity of the transplanted SCN may play a role in the recovery of circadian rhythmicity.

Importantly, the functional specificity retained in the selective transplantation of VP neurons from different regions of the fetal hypothalamus is strongly correlated with the distinguishing cytological and histochemical features of the grafts. Circadian functional capacity was evidenced only in those grafts of the presumptive SCN in which VP- and VIP-immunoreactive neurons were identified within SCN-like structures. The only SCN graft that failed to express a VP rhythm, or even consistently detectable levels of VP in the CSF, contained only a very few SCN-like cells but considerable numbers of other subtypes of VP neurons. Similarly, the inability of all grafts of the fetal PVN to generate rhythmic patterns of VP release was correlated with a cytoarchitectonic organization and a complement of neuropeptides that was not SCN-like but rather was homologous with the in situ PVN. In this regard, the functional anatomy of PVNderived grafts was distinguished by the presence of subtypes of VP neurons that projected principally to vasculature and exhibited no sign of association with neural perikarya immunoreactive for VIP. It is interesting that the presence of a few SCN-like parvicellular neurons in one PVN graft was not accompanied by circadian functional capacity, suggesting that either the ability of this component to generate a VP rhythm was masked by peptide release from the more numerous PVN-like VP neurons in the graft or a critical number of SCN neurons is required for the generation of a detectable VP rhythm.

In grafts of the entire fetal anterior hypothalamus, differences in the anatomical, morphological, and histochemical features of VP- and VIP-immunoreactive neurons within SCN-like and PVN-like cell groups have been reported to virtually mirror those that distinguish these hypothalamic nuclei in situ (Lehman et al., 1987; Wiegand and Gash, 1987, 1988a, b). For example, SCN-like and PVN-like vasopressinergic neurons exhibit pro- 
jections to distinct but appropriate targets in the host brain and differential associations with vascular elements within the transplants (Wiegand and Gash, 1988a, b). SCN-like VIP-containing neurons within fetal hypothalamic grafts have been reported to receive a specific and appropriate projection from neuropeptide $Y$-containing cells and, occasionally, the host retina (Lehman et al., 1987). Conversely, magnocellular vasopressinergic neurons, but not $\mathrm{SCN}$-like cell groups, receive a significant catecholaminergic projection from the host brain (Silverman and Sladek, 1988). In addition, neurons immunoreactive for oxytocin and corticotropin releasing factor have been identified in association with PVN-like aggregations of VP neurons, but not within SCN-like cell groups (Wiegand and Gash, 1987, 1988b). Preliminary immunohistochemical analyses of tissue obtained in the course of the present experiment indicate that anatomical and histochemical distinctions are maintained in a similar fashion in transplants of microdissected SCN and PVN (Wiegand et al., 1987).

The present experimental approach, whereby parameters related to the dissection of donor tissue are manipulated to selectively obtain cytologically distinct populations of neurons for transplantation, may provide a useful tool for examining the relationship between the function and neural organization of the SCN. Recent neural transplantation studies have utilized the entire fetal hypothalamus as donor tissue to study the restoration of circadian function in $\mathrm{SCN}$-lesioned hosts (DruckerColin et al., 1984; DeCoursey and Buggy, 1986; Lehman et al., 1987). In addition, neurons dcrived from fetal neocortex served as tissue controls for the hypothalamic grafts in these experiments. The present results suggest that it may be possible to correlate specific physiological indices of circadian function with distinctive anatomical features by transplanting discrete precursor populations of SCN neurons and by using neurochemically similar but cytologically distinct neurons derived from other developing brain regions as tissue controls.

\section{References}

Altman, J., and S. A. Bayer (1978a) Development of the diencephalon in the rat. I. Autoradiographic study of the time of origin and the settling patterns of neurons of the hypothalamus. J. Comp. Neurol. 182: 945-972.

Altman, J., and S. A. Bayer (1978b) Development of the diencephalon in the rat. II. Correlation of the embryonic development of the hypothalamus with the time of origin of its neurons. J. Comp. Neurol. 182: 972-994.

Boer, G. J., D. M. Gash, L. Dick, and N. Schluter (1985) Vasopressin neuron survival in neonatal Brattleboro rats: Critical factors in graft development and innervation of the host brain. Neuroscience 15: 1087-1 109 .

DeCoursey, P. J., and J. Ruggy (1986) Restoration of locomotor rhythmicity in SCN-lesioned golden hamsters by transplantation of the fetal SCN. Soc. Neurosci. Abstr. 12: 210.

Drucker-Colin, R., R. Aguilar-Roblero, F. Garcia-Hernandez, F. Fernandez-Cancino, and F. B. Rattoni (1984) Fetal suprachiasmatic nucleus transplants: Diurnal thythm recovery of lesioned rats. Brain Res. 311: 353-357.
Earnest, D. J., and C. S. Sladek (1987) Circadian vasopressin release from perifused rat suprachiasmatic explants in vitro: Effects of acute stimulation. Brain Res. 422: 398-402.

Gillette, M. U., and S. M. Reppert (1987) The hypothalamic suprachiasmatic nuclei: Circadian patterns of vasopressin secretion and neuronal activity in vitro. Brain Res. Bull. 19: 135-139.

Green, D. J., and R. Gillette (1982) Circadian rhythm of firing rate recorded from single cells in rat suprachiasmatic brain slice. Brain Res. 245: 198-200.

Inouye, S. T., and H. Kawamura (1982) Characteristics of a circadian pacemaker in the suprachiasmatic nucleus. J. Comp. Physiol. 146: 153-160.

Lehman, M. N., R. Silver, W. R. Gladstone, R. M. Kahn, M. Gibson, and E. L. Bittman (1987) Circadian rhythmicity restored by neural transplant. Immunocytochemical characterization of the graft and its integration with the host brain. J. Neurosci. 7: 1626-1638.

Moore, R. Y. (1983) Organization and function of a central nervous system circadian oscillator: The suprachiasmatic hypothalamic nucleus. Fed. Proc. 42: 2783-2789.

Paxinos, G., and C. Watson (1986) The Rat Brain in Stereotaxic Coordinates, Academic, Orlando.

Reppert, S. M., and W. J. Schwartz (1984) The suprachiasmatic nuclei of the fetal rat: Characterization of a functional circadian clock using ${ }^{14} \mathrm{C}$-labeled deoxyglucose. J. Neurosci. 4: 1677-1682.

Schwartz, W. J., and S. M. Reppert (1985) Neural regulation of the circadian vasopressin rhythm in cerebrospinal fluid: A pre-eminent role for the suprachiasmatic nuclei. J. Neurosci. 5: 2771-2778.

Schwartz, W. J., R. J. Coleman, and S. M. Reppert (1983) A daily vasopressin rhythm in rat cerebrospinal fluid. Brain Res. 263: 105112.

Silverman, W. F., and J. R. Sladek, Jr. (1988) Neural transplantation as a tool to examine vasopressin neuronal interactions. In Vasopressin: Cellular and Integrative Function, A. W. Cowley, J. F. Liard, and D. A. Ausiello, eds., pp. 281-288, Raven, Ncw York.

Sladek, C. S., M. L. Blair, Y. Chen, and R. W. Rockhold (1986) Vasopressin and renin response to plasma volume loss in spontaneously hypertensive rats. Am. J. Physiol. 250: $\mathrm{H} 443-\mathrm{H} 452$.

Sokol, H. W., E. A. Zimmerman, W. H. Sawyer, and A. G. Robinson (1976) The hypothalamic-neurohypophysial system of the rat: Localization and quantitation of neurophysin by light microscopic immunocytochemistry in normal rats and in Brattleboro rats deficient in vasopressin and a neurophysin. Endocrinology 98:1176-1188.

Vandesande, F., K. Dierickx, and J. De Mey (1975) Identification of vasopressin-neurophysin producing neurons of the rat suprachiasmatic nucleus. Cell Tissue Res. 156: 377-380.

Watson, R. E., S. J. Wiegand, R. W. Clough, and G. E. Hoffman (1986) The use of cryoprotectant to maintain long-term peptide immunoreactivity and tissue morphology. Peptides 7: 155-159.

Wiegand, S. J., and D. M. Gash (1987) Intraventricular transplants of anterior hypothalamus: Neurochemical and connectional specificity in morphologically distinct subtypes of neurophysin-containing neurons. Ann. N.Y. Acad. Sci. 495: 807-810.

Wiegand, S. J., and D. M. Gash (1988a) Characteristics of vasculature and neurovascular relations in intraventricular anterior hypothalamic transplants. Brain Res. Bull. 20: 105-124.

Wiegand, S. J., and D. M. Gash (1988b) Organization and efferent projections of transplanted suprachiasmatic nuclei. J. Comp. Neurol. 267: 562-579.

Wiegand, S. J., D. J. Earnest, and D. M. Gash (1987) Selective transplantation of the fetal suprachiasmatic and paraventricular nuclei: Stability and specificity in cytological and immunohistochemical characteristics. Proc. Soc. Neurosci. 13: 212. 